\title{
SEARCH FOR A NEW LIGHT GAUGE BOSON IN DECAYS OF $\pi^{0}$ AND $\eta$
}

C. Amsler ${ }^{15}$, D.S. Armstrong ${ }^{1, a}$, I. Augustin ${ }^{8, b}$, C.A. Baker ${ }^{5}$, B.M. Barnett ${ }^{11}$, C.J. Batty ${ }^{5}$, K. Beuchert ${ }^{2}$, P. Birien ${ }^{1}$, P. Blüm ${ }^{8}$, R. Bossingham ${ }^{1}$, K. Braune ${ }^{12}$, J. Brose ${ }^{11}$, D.V. Bugg ${ }^{9}$, M. Burchell ${ }^{6, c}$, T. Case ${ }^{1}$, A. Cooper ${ }^{9}$, K.M. Crowe ${ }^{1}$, T. Degener ${ }^{2}$, H.P. Dietz ${ }^{12}$,

S. von Dombrowski ${ }^{15}$, M. Doser ${ }^{6}$, W. Dünnweber ${ }^{12}$, D. Engelhardt ${ }^{8}$, M. Englert ${ }^{12}$, M.A. Faessler ${ }^{12}$, C. Felix ${ }^{12}$, R. Hackmann ${ }^{11}$, R.P. Haddock ${ }^{10}$, F.H. Heinsius ${ }^{1}$, N.P. Hessey ${ }^{7}$, P. Hidas ${ }^{4}$, P. Illinger ${ }^{12}$, D. Jamnik ${ }^{12, d}$, Z. Jávorfi ${ }^{4}$, H. Kalinowsky ${ }^{11}$, B. Kämmle ${ }^{7}$, T. Kiel ${ }^{8}$, J. Kisiel ${ }^{12, e}$, E. Klempt ${ }^{3}$, M. Kobel ${ }^{6, f}$, H. Koch ${ }^{2}$, C. Kolo ${ }^{12}$, K. Königsmann ${ }^{12, g}$, M. Kunze ${ }^{2}$, R. Landua ${ }^{6}$, F. Loser $^{7}$, J. Lüdemann ${ }^{2}$, H. Matthäy ${ }^{2}$, M. Merkel ${ }^{11, h}$, J.P. Merlo ${ }^{11}$, C.A. Meyer ${ }^{13}$, L. Montanet ${ }^{6}$, A. Noble ${ }^{15}$, F. Ould-Saada ${ }^{15}$, K. Peters ${ }^{2}$, C.N. Pinder ${ }^{5}$, G. Pinter ${ }^{4}$, S. Ravndal ${ }^{2}$, J. Salk ${ }^{2}$, A.H. Sanjari ${ }^{9, i}$, E. Schäfer ${ }^{11}$, B. Schmid ${ }^{15, j}$, P. Schmidt ${ }^{7}$, S. Spanier ${ }^{11}$, C. Straßburger ${ }^{3}$, U. Strohbusch ${ }^{7}$, M. Suffert ${ }^{14}$, D. Urner ${ }^{15}$, C. Völcker ${ }^{12}$, F. Walter ${ }^{11}$, D. Walther ${ }^{2}$, U. Wiedner ${ }^{7}$, N. Winter ${ }^{8}$, J. Zoll ${ }^{4}$, B. Zou ${ }^{9}$, C. Zupančič ${ }^{12}$

\section{Crystal Barrel Collaboration}

\author{
1 University of California, LBL, Berkeley, CA 94720, USA \\ ${ }^{2}$ Universität Bochum, D-44780 Bochum, Germany \\ ${ }^{3}$ Universität Bonn, D-53115 Bonn, Germany \\ 4 Academy of Science, H-1525 Budapest, Hungary \\ ${ }^{5}$ Rutherford Appleton Laboratory, Chilton, Didcot OX11, 0QX, UK \\ 6 CERN, CH-1211 Genève, Switzerland \\ 7 Universität Hamburg, D-22761 Hamburg, Germany \\ 8 Universität Karlsruhe, D-76344 Karlsruhe, Germany \\ ${ }^{9}$ Queen Mary and Westfield College, London E1 4NS, UK \\ 10 University of California, Los Angeles, CA 90024, USA \\ 11 Universität Mainz, D-55099 Mainz, Germany \\ 12 Universität München, D-85748 München, Germany \\ 13 Carnegie Mellon University, Pittsburgh, PA, USA \\ 14 Centre de Recherches Nucléaires, F-67037 Strasbourg, France \\ 15 Universität Zürich, CH-8001 Zürich, Switzerland
}

a) Now at College of William and Mary, Williamsburg, VA, USA

b) Now at University of Siegen, Siegen, Germany

c) Now at University of Kent, Canterbury, UK

d) On leave of absence from the University of Ljubljana, Ljubljana, Slovenia

e) On leave of absence from the University of Silesia, Katowice, Poland 
f) Now at University of Freiburg, Freiburg i. Br., Germany

g) Now at Max Planck Institut, Heidelberg, Germany

$h$ ) Now at CERN, Genève, Switzerland

i) Now at State University of New York, Stony Brook, NY, USA

j) Now at University of California, Irvine, CA, USA 


\begin{abstract}
We have searched for new light gauge bosons produced in $\pi^{0}$ and $\eta$ decays by studying the kinematically well-constrained reactions $\bar{p} p \rightarrow \pi^{0} \pi^{0} \pi^{0}$ and $\bar{p} p \rightarrow \pi^{0} \pi^{0} \eta$, where one $\pi^{0}$ or the $\eta$ decays through the emission of a single photon recoiling against a missing state $X$ (where $X$ is a long-lived weakly interacting particle or $X \rightarrow \nu \bar{\nu})$. No signal has been observed and branching ratio upper limits of $6 \times 10^{-5}$ at $90 \%$ C.L. have been obtained for masses of the gauge boson lying between $\sim 65 \mathrm{MeV}$ and $125 \mathrm{MeV}\left(\pi^{0}\right.$ decay), and $6 \times 10^{-5}$ at $90 \%$ C.L., for $X$ masses between $\sim 200 \mathrm{MeV}$ and $525 \mathrm{MeV}$ ( $\eta$ decay). The $\pi^{0}$-decay limit represents a factor of 4 to 8 improvement when compared to the existing limit, whereas the $\eta$ decay limit has been measured for the first time, thereby extending the $m_{X}$ range from $130 \mathrm{MeV}$ up to $525 \mathrm{MeV}$.
\end{abstract}

There exist various extensions of the Standard Model $(S U(3) \times S U(2) \times U(1))$ of strong and electroweak interactions. Some of them require an extra gauge group $U^{\prime}(1)$ and, hence, a new gauge boson $X$. This possibility is realised in grand unified theories [1], in supersymmetric theories [2], in superstring-inspired models [3] and in models including a new long-range interaction, the fifth force [4]. Some $X$ bosons could be light enough to be detected in rare or (Standard Model) forbidden decays of light mesons $(\pi, K, \eta, \ldots)[5,6]$. To look for such new light particles, with masses of the order of the pion mass and with small couplings, high rates and precision are needed. Constraints on branching ratios for the decay of a pseudoscalar ${ }^{1)}$ $P \rightarrow \gamma X, P$ being a $\pi^{0}$, an $\eta$ or an $\eta^{\prime}[6]$ are summarised in table 1 . Two possibilities are considered depending on the interaction of the $X$ boson: (I) $X$ interacts weakly with both leptons and quarks, in which case it decays in our detector $\left(P \rightarrow \gamma e^{+} e^{-}\right.$or $\left.P \rightarrow \gamma \nu \bar{\nu}\right)$. The decay $X \rightarrow \nu \bar{\nu}$ is experimentally more favourable to study since $X \rightarrow e^{+} e^{-}$is overwhelmed by the Dalitz decay $\pi^{0} \rightarrow \gamma e^{+} e^{-}$; (II) $X$ interacts weakly only with quarks and hence has a long lifetime and does not decay in our detector. In both cases the analysis consists of looking for a photon and a missing mass. The decay $X \rightarrow e^{+} e^{-}$is not considered in this work.

Consider the decay $P \rightarrow \gamma \gamma$. In the rest frame of the decaying pseudoscalar $P$, the photon energy is given by $E_{\gamma}^{*}=m_{P} / 2$, where $m_{P}$ is the mass of the pseudoscalar $P$. Because of detector inefficiencies, one of the two photons can be lost. This leads to a peak in the energy distribution with a width determined by the experimental resolution. If one replaces the missing photon by a missing massive gauge boson $X$, the photon energy distribution peaks at

$$
E_{\gamma}^{*}=\frac{m_{P}}{2}\left(1-\frac{m_{X}^{2}}{m_{P}^{2}}\right),
$$

where $m_{X}$ is the mass of the gauge boson $X$.

To look for a hypothetical gauge boson $X$ in the decays $\pi^{0} \rightarrow \gamma X$ or $\eta \rightarrow \gamma X$, we exploit the fact that the annihilation of antiprotons with protons is a rich source of mesons (e.g., $\left.\pi^{0}, \eta, \omega\right)$ and that the Crystal Barrel detector is ideally designed for measurements of final states with many photons. We use the well-constrained reactions $\bar{p} p \rightarrow \pi^{0} \pi^{0} \pi^{0}$ and $\bar{p} p \rightarrow \pi^{0} \pi^{0} \eta$ because of the relatively high branching ratios $B R\left(\bar{p} p \rightarrow \pi^{0} \pi^{0} \pi^{0}\right)=(0.63 \pm 0.10) \%[7]$ and

1) The advantage of pseudoscalar decays into $\gamma X$ over $K^{+} \rightarrow \pi^{+} X$ is the unambiguous theoretical interpretation: if there is a signal it can only be a spin-1 gauge boson [5]. 
$B R\left(\bar{p} p \rightarrow \pi^{0} \pi^{0} \eta\right)=(0.65 \pm 0.07) \%$ [8] at rest in liquid hydrogen. Before describing the data selection and analysis, we first summarise the capabilities of our detector.

The data have been collected using the Crystal Barrel detector on beam line $\mathrm{C} 2$ at the Low Energy Antiproton Ring (LEAR) at CERN. Antiprotons with a momentum of $200 \mathrm{MeV} / \mathrm{c}$ are stopped in a 4-cm-long liquid hydrogen target. A matrix of silicon counters in front of the target defines and monitors the incident $\bar{p}$ beam (typically $10^{4} \bar{p} / s$ ). The target is surrounded by two cylindrical proportional wire chambers (PWCs), a jet drift chamber (JDC) to detect charged particles, and a barrel-shaped calorimeter consisting of $1380 \mathrm{CsI}(\mathrm{Tl})$ crystals, 16 radiation lengths deep, with photodiode readout. The CsI calorimeter covers the polar angles between $12^{\circ}$ and $168^{\circ}$ with full coverage in azimuth. The useful acceptance for shower detection is $0.95 \times 4 \pi$ sr. The energy calibration is provided by $\pi^{0} \rightarrow 2 \gamma$ decays by imposing the mass of the $\pi^{0}$. The energy resolution, which varies slightly from run to run, is given by $\sigma_{E} / E_{\gamma} \sim 2.5 \% / \sqrt[4]{E_{\gamma} / G e V}$. The angular resolution (polar and azimuthal) $\sigma_{\theta, \phi}$ varies with the photon energy from $20 \mathrm{mrad}$ to $35 \mathrm{mrad}$ for isolated showers and is $\sim 35 \mathrm{mrad}$ for two or more showers when merged into a single cluster of crystals. The whole assembly is located in a solenoid magnet providing a homogeneous field of $1.5 \mathrm{~T}$ parallel to the incident $\bar{p}$ beam. A more detailed description of the detector, the trigger conditions and the reconstruction procedure is given in Ref. [9].

The analysis is based on $15 \times 10^{6}$ triggered all-neutral events. The triggering was performed using the PWCs and the inner layers of the JDC to veto charged tracks. Events with residual charged tracks are removed in the offline data selection. 1,019,634 events containing exactly 5 electromagnetic showers (PEDs) with energy deposits exceeding the threshold energy $E_{\text {cut }}=20$ $\mathrm{MeV}$ are selected. Events with a PED induced in the last crystal rows around the beam entrance and exit pipes are removed to suppress energy leakage. Shower fluctuations may produce fake photons, so-called split-offs. These are characterised by their small energy and their proximity to a higher energy PED. Such split-off events are suppressed by requiring the relative energy deposition of two neighbouring PEDs, the centroids of which are separated by less than $14^{\circ}$, to be larger than 0.18 . The next step is to reconstruct two $\pi^{0}$ 's out of the $5 \gamma$ sample $(818,655$ events). The invariant masses of all combinations of two photons are calculated and events containing two $\pi^{0}$ 's and a single $\gamma$ are kept. When more than one combination is found, only the best configuration with a confidence level C.L. $>2.7 \%$ is chosen. The surviving data sample consists of 187,145 events. Once the $\pi^{0} \pi^{0} \gamma$ events are selected, a kinematic fit with 3 constraints (3-C fit) $\left(\pi^{0} \pi^{0} P_{\text {miss }}\right.$, where $P_{\text {miss }}$ is a missing $\pi^{0}$ or a missing $\eta$ ) is performed, ignoring the remaining single photon. A confidence level greater than $15 \%$ is required. We are left with $95,392 \pi^{0} \pi^{0} \pi_{\text {miss }}^{0}$ and $17,943 \pi^{0} \pi^{0} \eta_{\text {miss }}$ fit events.

The method used to search for an $X$ signal now consists of plotting the energy $E_{\gamma}^{*}$ of the single $\gamma$ in the rest frame of $P_{\text {miss }}$. The measured energy and momentum of the fifth photon (which is ignored by the fitting procedure) and the fit values of $P_{\text {miss }}$ are used to calculate $E_{\gamma}^{*}$.

The background contribution can be split into two categories: $5 \gamma$ final states without and with missing energy and momentum. The first source of background comes from real $\pi^{0} \pi^{0} \gamma$ events, due to the finite resolution of the detector. Here it is mainly the channel $\bar{p} p \rightarrow \omega \pi^{0}$ $\left(\omega \rightarrow \pi^{0} \gamma\right.$ ) which feeds into the fit $\pi^{0} \pi^{0} \pi_{m i s s}^{0}$ and to a lesser extent into $\pi^{0} \pi^{0} \eta_{\text {miss }}$. To reduce this background the selected $5 \gamma$ data events are kinematically fit to the following final states: $\bar{p} p \rightarrow \pi^{0} \omega\left(\omega \rightarrow \pi^{0} \gamma\right)(7-\mathrm{C}$ fit $), \bar{p} p \rightarrow \eta \omega\left(\omega \rightarrow \pi^{0} \gamma\right.$ and $\left.\eta \rightarrow \gamma \gamma\right)$ (7-C fit), $\bar{p} p \rightarrow \pi^{0} \eta \gamma(6-\mathrm{C}$ 
fit) and $\bar{p} p \rightarrow \eta \eta \gamma$ (6-C fit). If one of the $5 \gamma$ combinations fits one of the above final states with a C.L. $>1 \%$, the event is rejected. About $25 \%$ of events are rejected for $\pi^{0} \pi^{0} \pi_{m i s s}^{0}$, while the proportion of rejected events is negligible in the case of $\pi^{0} \pi^{0} \eta_{\text {miss }}$. The second source of background consists of the following final states: $\bar{p} p \rightarrow \pi^{0} \pi^{0} \pi^{0}$, where one photon from a $\pi^{0}$ is undetected and $\bar{p} p \rightarrow \pi^{0} \pi^{0} \eta$, where one photon from the $\eta$ is undetected. There are two main ways in which a photon can escape detection. Either the $\gamma$ is lost around the beam entrance or exit pipes $\left(\theta \geq 168^{\circ}\right.$ or $\left.\theta \leq 12^{\circ}\right)$ or between the two halves of the barrel $\left(\theta \sim 90^{\circ}\right)$ or, alternatively, its energy is below the detection threshold of $20 \mathrm{MeV}$. In order to reduce the missing photon contribution, an event is discarded if $\left|\cos \left(\theta_{\gamma_{\text {miss }}}\right)\right| \geq 0.95$ or $\left|\cos \left(\theta_{\gamma_{\text {miss }}}\right)\right| \leq$ 0.02. We are left with $48,158 \pi^{0} \pi^{0} \pi_{\text {miss }}^{0}$ and $10,827 \pi^{0} \pi^{0} \eta_{\text {miss }}$ events.

For the $\eta$ decay we also take into account the channel $\bar{p} p \rightarrow K_{S} K_{L}$, where $K_{S} \rightarrow \pi^{0} \pi^{0}$ and $K_{L}$ interacts in the barrel and produces one PED. This background is relevant since the mass of $K_{L}\left(497.67 \mathrm{MeV} / \mathrm{c}^{2}\right)$ is close to the $\eta$ mass. The $K_{L}$ and $K_{S}$ emerge back to back and therefore events are rejected for which the invariant mass ${ }^{2}$ of the two $\pi^{0} \mathrm{~s}$ is in the region $455 \leq M_{\pi^{0} \pi^{0}} \leq 506 \mathrm{MeV} / \mathrm{c}^{2}$ and the angle between the $K_{S}$ direction and the fifth photon is greater than $167^{\circ}$. About $50 \%$ of the events far from the $\eta \rightarrow \gamma \gamma$ peak are rejected. This reduces the $\pi^{0} \pi^{0} \eta_{\text {miss }}$ sample to 8,961 events.

The remaining background events tend to accumulate along the line of maximum possible $\gamma$ energy in the laboratory, $E_{\gamma}^{l a b}$, in a two dimensional plot $E_{\gamma}^{l a b}$ vs. $E_{\gamma}^{*}$. This happens for events where the fifth photon has the same direction as $P_{\text {miss }}$. This is the case for the small fraction of $\pi^{0} \pi^{0} \gamma$ events $\left(K_{L} K_{S}, \omega \pi^{0}, \ldots\right)$ that have not been removed by the above cuts. The following cuts are therefore applied: $E_{\gamma}^{\text {lab }} / E_{\gamma}^{*} \leq 2.6$ for $\eta$ decay and $E_{\gamma}^{\text {lab }} / E_{\gamma}^{*} \leq 10.0$ for $\pi^{0}$ decay. About $30 \%$ of the remaining background events are rejected by these cuts, without affecting the signal. The final sample consists of $24,503 \pi^{0} \pi^{0} \pi_{\text {miss }}^{0}$ and $5,121 \pi^{0} \pi^{0} \eta_{\text {miss }}$ events.

The next step is to check the method and determine the acceptance and energy resolution. A Monte Carlo program, based on the GEANT [10] package, which includes our detector geometry, is used to generate and simulate the various $\bar{p} p$ final states for both signal and background events. The simulated events are then passed through the same reconstruction and analysis chain as the real data. In the generation of signal events it is assumed that the particle $X$ does not interact in the detector. Its mass is varied between 0 and $m_{P}$. In the $\pi^{0} \pi^{0} \pi^{0}$ generated sample, one $\pi^{0}$ is forced to decay into $\gamma X$. For $m_{X} \leq 65 \mathrm{MeV} / \mathrm{c}^{2}$ (corresponding to high $\gamma$ energies) the missing photon background $\left(E_{\gamma}^{*} \sim m_{\pi^{0}} c^{2} / 2\right)$ is dominant. At higher mass range (smaller $\gamma$ energy) the sensitivity is limited by the detection threshold for the photon (20 $\mathrm{MeV}$ in the laboratory frame). The detector is therefore most sensitive to $m_{X}$ between $\sim 65$ and $\sim 125 \mathrm{MeV} / \mathrm{c}^{2}$ for $\pi^{0}$ decay. With the $\eta$ decay we can investigate higher $X$ masses between $\sim 100$ and $\sim 525 \mathrm{MeV} / \mathrm{c}^{2}$.

The photon energy resolution varies between $\sigma=(4.0-5.0) \mathrm{MeV}$ and $\sigma=(3.5-4.5) \mathrm{MeV}$, depending on $m_{X}$, for $\pi^{0}$ and $\eta$ decay, respectively. On the $\pi^{0} \rightarrow \gamma \gamma$ peak the resolution is about $10 \mathrm{MeV}$. The lower and upper values correspond to the upper and lower limits, respectively, for $m_{X}$ given above.

Important background sources are also simulated: (i) $\bar{p} p \rightarrow \pi^{0} \omega,\left(\omega \rightarrow \pi^{0} \gamma\right)$, (ii) $\bar{p} p \rightarrow$ $\pi^{0} \pi^{0} \pi^{0}\left(\pi^{0} \rightarrow \gamma \gamma\right)$, and (iii) $\bar{p} p \rightarrow \pi^{0} \pi^{0} \eta\left(\pi^{0}, \eta \rightarrow \gamma \gamma\right)$. We find that the $5 \gamma$ final state $\pi^{0} \omega$

2) The $\pi^{0} \pi^{0}$ mass used $(\sim 480)$ is lower than the nominal $K_{S}$ mass (497.671) because of the decay length of $K_{S}$. The $K_{S}$ vertex is not, as assumed, in the centre of the target. 
and the $6 \gamma$ final state $\pi^{0} \pi^{0} \pi^{0}$ (where one photon from a $\pi^{0}$ is not reconstructed) together reproduce the shape of the data. The normalisation is given by the known branching ratios: $B R\left(\bar{p} p \rightarrow \pi^{0} \pi^{0} \pi^{0}\right)=(0.63 \pm 0.10) \%, B R\left(\bar{p} p \rightarrow \pi^{0} \omega\right)=(0.57 \pm 0.05) \%$ [11] and $B R(\omega \rightarrow$ $\left.\pi^{0} \gamma\right)=(8.5 \pm 0.5 \%)[12]$ and the Monte Carlo reconstruction efficiencies. Other final states, like $\pi^{0} \eta \gamma, \eta \eta \gamma, \omega \pi^{0} \pi^{0}$ (where two photons are not reconstructed), and $\omega \omega, \omega \rightarrow \pi^{0} \gamma$ (where one photon is not reconstructed), give a smaller contribution.

The signal events (with $P \rightarrow \gamma X$ ) are used to calculate the ratio of the number of simulated and accepted events to the number of generated events. The following efficiencies are obtained: $\epsilon_{\pi^{0}}=(11 \pm 1) \%$ for the $\pi^{0}$ decay and $\epsilon_{\eta}=(16 \pm 1) \%$ for the $\eta$ decay. The uncertainties include the dependence of the efficiency on $m_{X}$. The number of expected events for a given branching ratio $B R(X)=B R(P \rightarrow \gamma X)$ is $N_{X}=N_{\bar{p}} \times B R\left(\bar{p} p \rightarrow \pi^{0} \pi^{0} P\right) \times B R(X) \times \epsilon$. For the $3 \pi^{0}$ final state, a multiplicative factor 3 is included, to allow for the possible decay of any of the three $\pi^{0}$ s. The $15 \times 10^{6}$ analysed 0 -prong events correspond to $N_{\bar{p}}=(360 \pm 10) \times 10^{6}$ annihilations at rest. This has been determined by reconstructing annihilation events from $\bar{p} p \rightarrow \pi^{0} \pi^{0}$ and by using the absolute branching ratio $B R\left(\bar{p} p \rightarrow \pi^{0} \pi^{0}\right)$ [11]. We then expect $N_{X}^{\pi^{0}}=76 \pm 11$ and $N_{X}^{\eta}=35 \pm 4$ signal events for a branching ratio $B R(X)$ of $10^{-4}$.

After having discussed the background reduction and the Monte Carlo simulation we now present the data. First we discuss the $\pi^{0}$ decay.

The energy of the single $\gamma$ in the $\pi^{0}$ rest frame is shown in fig. 1 . A ten-parameter fit to the data includes the sum of three gaussians and a constant background term. The three gaussians have maxima around $m_{\pi^{0}} c^{2} / 2$ (at $69.2,69.7$ and $72.0 \mathrm{MeV}$ ). These peak positions and the resolutions $\left(4.2,10.3\right.$ and $26.0 \mathrm{MeV}$ ) are in agreement with what we expect for $\pi^{0} \omega$ events (no missing photon), $\pi^{0} \pi^{0} \pi^{0}$ events (where one photon is missing), and other smaller background sources like $\omega \pi^{0} \pi^{0}$ (where 2 photons are missing). The third gaussian is, as expected, wider than the first two and its amplitude much smaller (32 events/0.5 MeV compared to 187 for the first and 263 for the second gaussian). The background term is due to events where the fifth photon found does not belong to the missing $\pi^{0}$. The global fit shows that the spectrum is well understood.

The amplitudes are consistent with Monte Carlo predictions using the experimental branching ratios. To look for a signal various fits have been performed, with similar results:

- A gaussian with two free parameters (position and number of events) is fit to the data in the region between $E_{\gamma}^{*}=10$ and $67.5 \mathrm{MeV}$. The width is fixed to the experimental resolution. The 10 parameters of the fit described above are allowed to vary within the errors.

- A signal (two parameters) and a polynomial background (three parameters) are fit to the data far from the $\pi^{0} \rightarrow \gamma \gamma$ peak.

- A signal (two parameters), a polynomial background (three parameters), and two gaussians describing the $\pi^{0} \rightarrow \gamma \gamma$ peak (six parameters) are fit to the data between $E_{\gamma}^{*}=10$ and $67.5 \mathrm{MeV}$.

An upper limit of $B R\left(\pi^{0} \rightarrow \gamma X\right)<6 \times 10^{-5}$ is obtained at $90 \%$ C.L. for $m_{X}$ lying between $65 \mathrm{MeV} / \mathrm{c}^{2}$ and $125 \mathrm{MeV} / \mathrm{c}^{2}$. Near $m_{X}=0$ we obtain $B R\left(\pi^{0} \rightarrow \gamma X\right)<2.8 \times 10^{-4}$. A signal corresponding to $B R\left(\pi^{0} \rightarrow \gamma X\right)=5 \times 10^{-4}$, the existing direct measurement [13] ${ }^{3)}$, is also

3) In [14] results from $\pi^{0} \rightarrow \gamma X$ with $X \rightarrow e^{+} e^{-}$a decay mode not considered in this work are given.

The results from $\pi^{0} \rightarrow \nu \bar{\nu}[15]$ have been interpreted as giving a limit of $6.2 \times 10^{-6}$ on $B R\left(\pi^{0} \rightarrow \gamma \nu \bar{\nu}\right)$ [6]. 
indicated in fig. 1. The upper limit as a function of $m_{X}$ is shown in fig. 3(a).

Next we discuss the $\eta$ decay. The energy of the single $\gamma$ in the $\eta$ rest frame shows a flatter and lower background than for $\pi^{0}$ (fig. 2). The main reason is the reduced contribution from $6 \gamma$ events $\left(\pi^{0} \pi^{0} \eta\right.$, where one photon from the $\eta$ is lost). This is due to the smaller BR for $\eta \rightarrow \gamma \gamma$, the higher $\gamma$ efficiency for the $\eta$ decay and the lower combinatoric background (one for $\pi^{0} \pi^{0} \eta$ vs. three for $\pi^{0} \pi^{0} \pi^{0}$ ). In fig. 2 a six-parameter fit to the data is performed which includes one gaussian and a background term (second-order polynomial). No significant signal is found on top of the background curve. An upper limit of $B R(\eta \rightarrow \gamma X)<6 \times 10^{-5}$ is obtained at $90 \%$ C.L. for masses of the $X$ boson between $200 \mathrm{MeV} / \mathrm{c}^{2}$ and $525 \mathrm{MeV} / \mathrm{c}^{2}$. A weaker upper limit of $B R(\eta \rightarrow \gamma X)<3 \times 10^{-4}$ is obtained down to $m_{X} \sim 50 \mathrm{MeV}$. The upper limit as a function of mass is given in fig. 3(b). A signal corresponding to $B R(\eta \rightarrow \gamma X)=5 \times 10^{-4}$ is also indicated in fig. 2 (dashed curve).

To summarise, we have searched for new light gauge bosons produced in $\pi^{0}$ and $\eta$ decays by studying the kinematically well-constrained reactions $\bar{p} p \rightarrow \pi^{0} \pi^{0} \pi^{0}$ and $\bar{p} p \rightarrow \pi^{0} \pi^{0} \eta$, where one $\pi^{0}$ (in the first case) or the $\eta$ (in the second) decays through the emission of a single photon recoiling against a missing state $X$ (where $X$ is a long-lived weakly interacting particle or $X \rightarrow \nu \bar{\nu})$. The different sources of background are well understood. We have analysed $15 \times 10^{6}$ 0 -prong triggered events. No signal has been observed and branching ratio upper limits of $6 \times 10^{-5}$ at $90 \%$ C.L. have been obtained for $m_{X}$ in the range between $65 \mathrm{MeV}$ and $125 \mathrm{MeV}$ $\left(\pi^{0}\right.$ decay), and of $6 \times 10^{-5}$ at $90 \%$ C.L., for $X$ masses lying between $200 \mathrm{MeV}$ and $525 \mathrm{MeV}$ ( $\eta$ decay). A less stringent upper limit of $B R(P \rightarrow \gamma X)<3 \times 10^{-4}$ is obtained for the mass regions $0 \leq m_{X} \leq 65 \mathrm{MeV}\left(\pi^{0}\right.$ decay) and $50 \leq m_{X} \leq 200 \mathrm{MeV}$ ( $\eta$ decay). The $\pi^{0}$-decay limit improves the existing limit [13] by a factor of 4 to 8 , whereas the $\eta$ upper limit has been measured for the first time, thereby extending the $m_{X}$ range from $130 \mathrm{MeV}$ up to $525 \mathrm{MeV}$.

We would like to thank the technical staff of the LEAR machine group and of all the participating institutions for their invaluable contributions to the success of the experiment. We acknowledge financial support from the German Bundesministerium für Forschung und Technologie, the Schweizerischer Nationalfonds, the British Science and Engineering Research Council and the U.S. Department of Energy (contract No. DE-FG03-87ER40323 and DE-AC0376SF00098). F.H. Heinsius acknowledges support from the A. von Humboldt Foundation. We thank Dr. S. Gninenko who first brought this research subject to our attention.

\section{References}

[1] P. Langacker, Phys. Rep. 72C(1981)185.

[2] P. Fayet, Phys. Lett. 69B(1977)489; Nucl. Phys. B187(1981)184; S. Weinberg, Phys. Rev. D26(1982)287.

[3] J. Ellis et al., Nucl. Phys. B276(1986)14; J. Schwarz, Superstrings: the First 15 Years, World Sc. Pub., Singapore (1985).

[4] S. Glashow, Proc. Conf. Rencontres de Moriond, (1986).

[5] M.I. Dobroliubov and A.Yu. Ignatiev, Nucl. Phys. B309(1988)655.

[6] M.I. Dobroliubov, Yad. Fiz. 52(1990)551. [Sov. J. Nucl. Phys. 52(1990)352].

[7] C. Amsler et al., to be published in Phys. Lett.

This, however, is not a direct measurement. 
[8] C. Amsler et al., Phys. Lett. B322(1994)431.

[9] E. Aker et al., Nucl. Instrum. Methods A321(1992)69.

[10] R. Brun et al., Internal Report CERN DD/EE/84-1, CERN 1987.

[11] C. Amsler et al., Z. Phys. C58(1993)175.

[12] Review of Particle Properties, Phys. Rev. D45(1992).

[13] M. S. Atiya et al., Phys. Rev. Lett. 69(1992)733.

[14] R. Meijer Drees et al., Phys. Rev. Lett. 68(1992)3845

[15] C.M. Hoffman, Phys. Lett. B208(1988)149.

\begin{tabular}{|l|c|c|}
\hline Decay & $I$ & $I I$ \\
\hline$\pi^{0} \rightarrow \gamma X$ & $6.2 \times 10^{-6}$ & $2.7 \times 10^{-3}$ \\
$\eta \rightarrow \gamma X$ & $1.6-3.8 \times 10^{-4}$ & $2.0-4.7 \times 10^{-4}$ \\
$\eta^{\prime} \rightarrow \gamma X$ & $0.7-5.7 \times 10^{-6}$ & $0.3-2.3 \times 10^{-6}$ \\
\hline
\end{tabular}

Table 1: Upper limits for the decay of a pseudoscalar into a photon and a gauge boson $X[6]$. Two cases are considered: (I) $X$ interacts with leptons and quarks, (II) $X$ interacts only with quarks. The $\pi^{0}$-decay limit (case $I$ ) is based on a measured value obtained for $\pi^{0} \rightarrow \nu \bar{\nu}[15]$. 


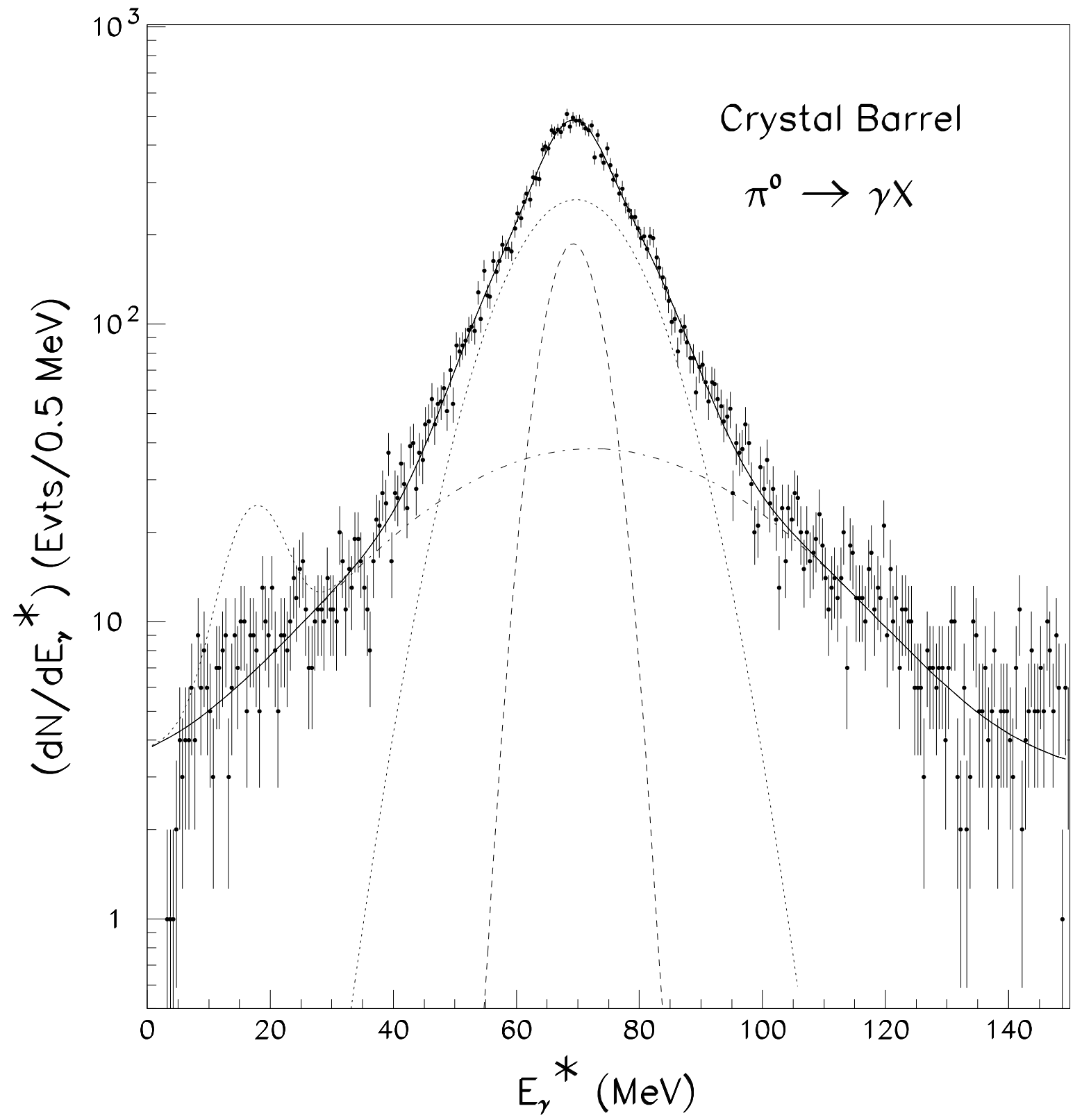

Figure 1: Energy of the single $\gamma$ in the $\pi_{\text {miss }}^{0}$ rest frame for the $\pi^{0} \pi^{0} \pi_{\text {miss }}^{0}$ kinematically fit sample after background suppression. The different contributions to the ten-parameter fit (full line) described in the text are shown separately: a narrow gaussian corresponding to $\pi^{0} \omega$ (dashed line), a broader gaussian corresponding to $\pi^{0} \pi^{0} \pi^{0}$ (finely dotted line), a much broader gaussian corresponding to other final states (like $\pi^{0} \pi^{0} \omega$ ), where two photons are lost (dashed-dotted line) on top of a constant background. A signal corresponding to a branching ratio for $\pi^{0} \rightarrow \gamma X$ with $m_{X}=120 \mathrm{MeV} / \mathrm{c}^{2}$ of $5 \times 10^{-4}$ is also drawn (dotted line). 


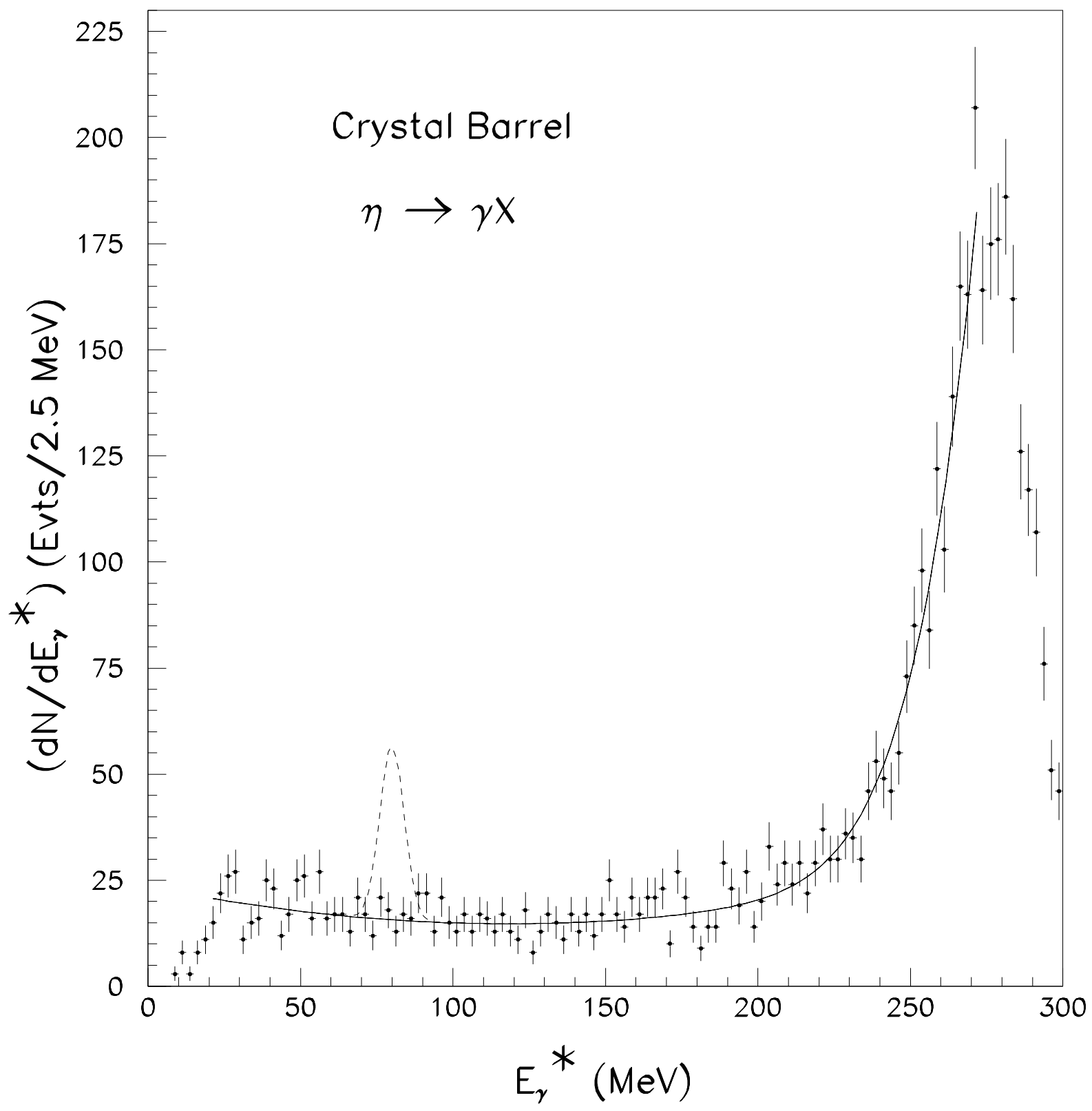

Figure 2: Energy of the single $\gamma$ in the $\eta_{\text {miss }}$ rest frame for the $\pi^{0} \pi^{0} \eta_{\text {miss }}$ kinematically fit sample after background suppression. The full curve corresponds to a six-parameter fit to the data (gaussian $+2^{\text {nd }}$ order polynomial). A signal corresponding to a branching ratio for $\eta \rightarrow \gamma X$ with $m_{X}=460 \mathrm{MeV} / \mathrm{c}^{2}$ of $5 \times 10^{-4}$ is also indicated (dashed line). 


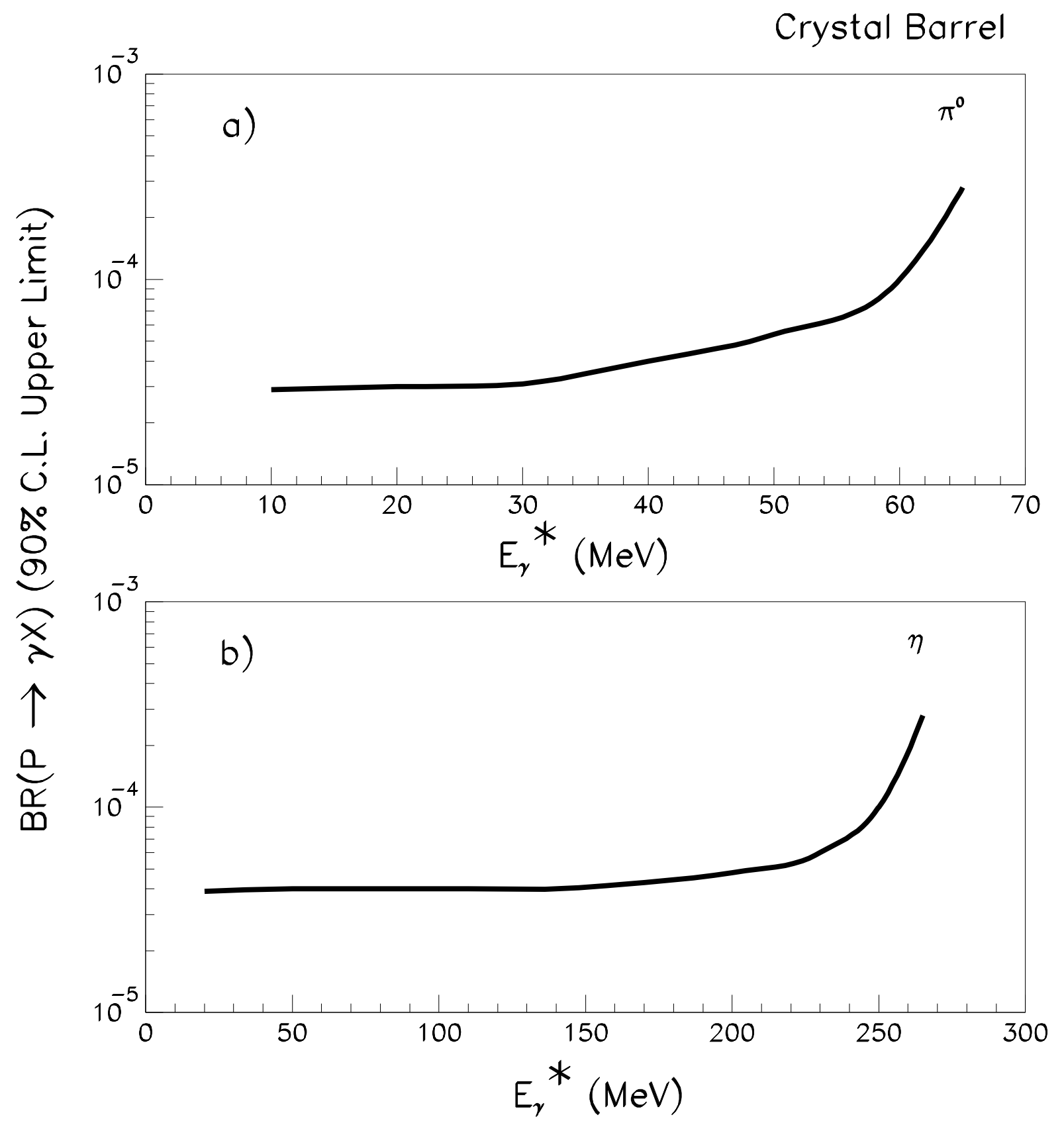

Figure 3: Branching ratio upper limit for the decays $\pi^{0} \rightarrow \gamma X$ (a) and $\eta \rightarrow \gamma X$ (b) as function of $E_{\gamma}^{*}$. 